\title{
Correction to: Prepare for the Pediatric Emergency Medicine Board Examination
}

Muhammad Waseem, Isabel A. Barata, Jennifer H. Chao, David Foster, and Noah Kondamudi

\section{Correction to:}

M. Waseem et al. (eds.), Prepare for the Pediatric Emergency Medicine Board Examination https://doi.org/10.1007/978-3-030-28372-8

This book was inadvertently published with the incorrect city and country on the affiliation of Dr. Noah Kondamudi. This has now been corrected in the book. 